\title{
Plan de gestión de costos en dirección de proyectos. Aplicación en una empresa deI sector minero-industrial de Colombia
}

FECHA DE RECEPCIÓN: 8 de marzo FECHA DE APROBACIÓN: abril 20 Pp. 22-39

Hugo Fernando Castro Silva*

H. Mauricio Diez-Silva **

Leonardo Fabio Quijano Brand ${ }^{* * *}$

Cost Management Plan in Project Management. Application in a mining-industrial company in Colombia

Plan de gestion des caûts de projets. Application dans une entreprise industrielle du secteur minier culombien

Plañ de gestãu de custos em direçãa de projetos. Aplicação em uma empresa do setor mineiro-industrial da Colômbia

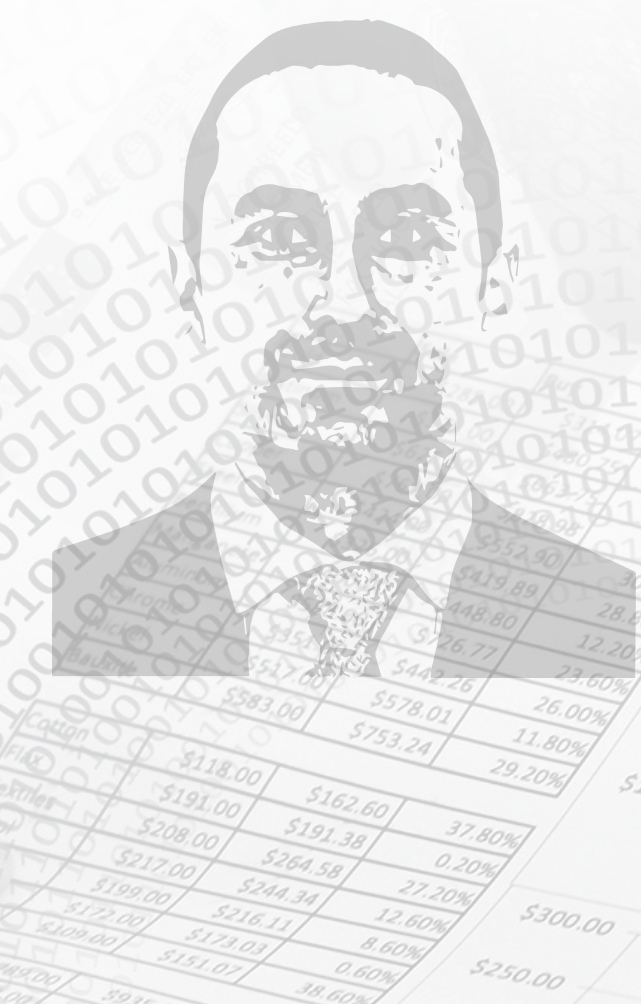

"Mágister en Administración de ProyectosUniversidad de Cooperación Internacional, San Fosé de Costa Rica, Mágister en Ingeniería Industrial- Universidad de Los Andes, Ingeniero Industrial- Universidad Pedagógica y Tecnológica de Colombia,Tunja.

*Doctor en Dirección de Proyectos de la Universidad Pública de Navarra, $M B A$, Universidad Politécnica de Madrid, Especialista en Administración, Universidad EAN, Especialista en Gerencia de Proyectos, Universidad de La Salle, Ingeniero de Diseño, Universidad de La Salle

*** Mágister en Dirección y Administración de Empresas, ISEAD Business Scholl, Especialización en Salud Ocupacional y Prevención de Riesgos Laborales, Universidad Pedagógica y Tecnológica de Colombia-Seccional Sogamoso, Administrador de Empresas, Universidad Pedagógica y Tecnológica de Colombia. 


\section{RESUMEN}

En este artículo se analiza la aplicación de prácticas de gestión de costos en dirección de proyectos en una empresa del sector minero-industrial de Colombia. Se han adaptado procesos del estándar internacional del Project Management Institute (PMI), para posteriormente ser aplicados en un proyecto de una empresa especializada en el diseño y fabricación de estructuras metálicas, montajes mecánicos y servicios de mantenimiento.

Se ha llevado a cabo un estudio de investigación aplicada para proponer y adaptar un método de trabajo para la gestión de costos en proyectos del sector bajo estudio. Además, se ha utilizado la acción-participación para realizar la validación de la propuesta en el proyecto seleccionado. Se han analizado los resultados obtenidos para realizar mejoras al método y proponer líneas de trabajo futuro.

Con este estudio se pretende ilustrar las ventajas de aplicar mejores prácticas de dirección de proyectos en casos de aplicación real. Se ha encontrado que la aplicación del método ha generado mejoras en los beneficios de la empresa, y que a partir de un proceso de perfeccionamiento y adaptación futura, su estructura puede ser aplicable en otras empresas que pertenecen al mismo sector.

\section{ABSTRACT}

In this article, cost management practices application in project management is analyzed in a company from the mining industrial sector. International standard processes from the Project Management Institute have been adapted to further apply them in a company project specialized in designing and manufacturing metal frames, mechanical mounts and maintenance services.

An applied research project has been carried out to propose and adapt a work method for cost management in projects belonging to this sector. Besides the action-participation method has been used to prove the validity of this kind of project. Results have been analyzed to improve the method and to propose guidelines for future works.

Through this study, we show the advantages of applying better practices of project management in real cases. The application of this method has brought great improvements in the company, and starting from a permanent improvement and adaptation process, its frame can be applied to other companies belonging to the same economic sector.

\section{Palabras claves}

Gestión de costos

Presupuesto

Dirección de proyectos

Sector minero-industrial Planeación
Key words

Cost management Budget

Project management Mining-industrial sector Planning 


\section{RESUMÉÉ}

Cet article analyse la mise en place de pratique de gestion des coûts de projets dans une entreprise industrielle colombienne du secteur minier. Différents processus standardisés et internationaux du Project Management Institute (PMI) y ont été adaptés pour être postérieurement appliqué au projet d'amélioration d'une entreprise spécialisée dans la création et la fabrication de structures métalliques, mécaniques et de services de maintenance.

Une investigation ayant comme objectif de proposer et d'adapter une méthode de travail pour la gestion des coûts de projets dans le secteur étudié a été entreprise; et un schéma d'action-participation utilisé pour valider la proposition du projet sélectionné.

Les résultats obtenus ont été analysés en vue d'adapter la méthode et d'en proposer des améliorations pour de futurs projets. L'objectif de cette étude est de montrer les avantages réels d'une meilleure mise en cuvre des pratiques de gestion de projet. L'étude a montré que l'application de la méthode a généré de réels bénéfices pour l'entreprise et que, par un processus de perfectionnement et d'adaptation, sa structure est applicable à d'autres entreprises du même secteur d'activité.

\section{RESUMO}

Neste artigo analisa-se a aplicação de práticas de gestão de custos em direção de projetos em uma empresa do setor mineiro-industrial da Colômbia. Adaptaram-se processos do standard internacional do Project Management Institute (PMI), a serem aplicados posteriormente em um projeto de uma empresa especializada em desenho e fabricação de estruturas metálicas, montagens mecânicas e serviços de manutenção.

Um estudo de pesquisa aplicada foi feito para propor e adaptar um método de trabalho para a gestão de custos em projetos do setor em estudo. Além, usou-se a ação-participação para realizar a validação da proposta no projeto selecionado. Analisaram-se os resultados obtidos para fazer melhoras ao método e propor linhas de trabalho futuro.

Com este estudo pretende-se mostrar as vantagens de fazer melhores práticas de direção de projetos em casos de aplicação real. Encontrou-se que a aplicação do método tem gerado melhoras nos benefícios da empresa, e que, a partir de um processo de aperfeiçoamento e adaptação futura, sua estrutura pode ser aplicável em outras empresas que pertençam ao mesmo setor.

\section{Mots clefs}

Gestion des coûts

Budget

Gestion de projets

Secteur minier et industriel

\section{Palavras-chave}

Gestão de custos

Pressuposto

Direção de projetos

Setor mineiro-industrial, Planejamento 


\section{INTRODUCCIón}

$\mathrm{P}$ ara la adopción de prácticas de dirección de proyectos en las organizaciones se utilizan diversos instrumentos y estrategias que han evolucionado a medida que se ha profesionalizado el Project Management, que desde los años sesenta y setenta se ha difundido y desarrollado por el trabajo de asociaciones profesionales como el Project Management Institute (PMI) y la Association for Project Management (APM), entre otras (Morris, P. W. G., et al., 2006). Según Morris (2006), se puede apreciar que los cuerpos de conocimiento contienen directrices y mejores prácticas que permiten perfeccionar las competencias, la formación y los procesos de gestión de los proyectos.

En ese sentido, la creciente aceptación de la dirección de proyectos indica que la aplicación de conocimientos, procesos, habilidades, herramientas y técnicas puede tener un impacto considerable en el éxito de los proyectos (Project Management Institute PMBOKR, 2012). Por tanto, la aplicación de dichos instrumentos en las organizaciones actuales, explorando su efectividad en diversos sectores de la economía, es el reto de los profesionales especializados en esta nueva área del conocimiento.

Dentro de ese contexto, diversos estudios han investigado la aplicación y difusión de prácticas de dirección de proyectos en países, sectores y organizaciones (Abbasi y AlMharmah, 2000; Ahlemann, et al., 2009; Bryde, D. J., 2003; Fortune, et al., 2011; Ika, et al., 2010; Muriithi y Crawford, 2003; Murphy y Ledwith, 2007; White y Fortune, 2002), y otros estudios han comparado sus estructuras (MontesGuerra, et al., 2011; Morris, P. W. G., et al., 2006; Wirth y Tryloff, 1995). En general se puede apreciar que la puesta en práctica de la dirección de proyectos se ha hecho habitualmente con la orientación de algún BOK o estándar, la implementación de una metodología, y el uso de técnicas y herramientas (Shenhar y Dvir, 2007; White y Fortune, 2002).

Dentro de las áreas de conocimiento de la dirección de proyectos (Project Management Institute PMBOKR 2012), la gestión de costos es precisamente una de las que mayor cantidad de técnicas y herramientas aporta para mejorar los procesos de ejecución de estos. Esta área trabaja, principalmente, sobre el costo de los recursos necesarios para completar las actividades del proyecto, teniendo en cuenta el efecto de las decisiones en cuanto a los costos recurrentes subsecuentes de utilizar, mantener y apoyar el producto, servicio o resultado del proyecto.

Dentro del trabajo de gestión de costos, el PMI plantea tres procesos esenciales que han sido aplicados en el desarrollo de este trabajo de investigación: la estimación de costos, la determinación del presupuesto y el control del costo (Project Management Institute PMBOKR, 2012). Estimar los costos es

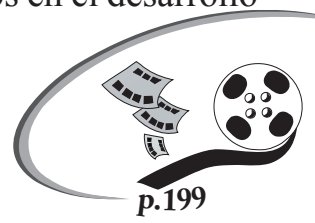
el proceso que consiste en desarrollar una aproximación de los recursos monetarios necesarios para completar las actividades del proyecto.

La determinación del presupuesto consiste en sumar los costos estimados de paquetes de trabajo para establecer la línea base de costo autorizada. Por último, el control del costo es el proceso con el que se monitorea la situación del proyecto para actualizar el presupuesto del mismo y gestionar cambios a la línea base del costo, es decir, al presupuesto inicial.

Por otra parte, uno de los sectores en los cuales la aplicación del área de conocimiento de gestión de costos se hace esencial, es en un campo tan competitivo como el industrial. En dicho sector se hace necesario estandarizar y caracterizar los procesos, crear estructuras de costos y financieras, realizar planes de gestión, controlar la trazabilidad de las etapas de los procesos, de la transformación de los materiales, de las horas hombre de trabajo, de los avances de las obras y, en general, del marco global de cada uno de los proyectos que emprenda una organización, de manera que brinden la información más completa y precisa a la hora de evaluar una nueva oportunidad de negocio con un cliente.

Sobre la base de lo planteado, se considera que el éxito en la aplicación de procesos de gestión de costos en un entorno en concreto, debe seguir la ruta de trabajo descrita en los párrafos anteriores, donde se plantea, según la bibliografía, 
que la aplicación y adaptación de los procesos de gestión de proyectos debe hacerse siguiendo la orientación de algún BOK o estándar, además de implementar un método y usar técnicas y herramientas. Por lo tanto, se ha utilizado la gestión de costos y el sector industrial colombiano para evaluar la aplicación de un método adaptado a partir del estándar del PMI y aplicado en actividades del sector mencionado.

Con el desarrollo de este trabajo de investigación se pretende mostrar las ventajas de aplicar mejores prácticas de dirección de proyectos en entornos reales, definiendo y utilizando una estructura para el plan de gestión de costos de un proyecto adaptado a partir de un estándar internacional. Se ha procurado que el proceso aplicado permita controlar el costo durante toda la ejecución del proyecto, generando beneficios en materia de involucrados, costo, tiempo y alcance, además de forjar líneas de trabajo futuro en el área de conocimiento del estudio.

El documento que se presenta se ha estructurado en seis partes. La primera, muestra la introducción del trabajo, realizada a partir del marco teórico principal para el estudio. En la segunda, se presenta la metodología utilizada, que hace referencia a la forma como se adaptó el estándar internacional sobre el proyecto estudiado. La tercera, describe la contextualización del sector y la empresa analizada. La cuarta, indica la forma como se aplicó la gestión de costos en el proyecto elegido. En la quinta, se documentan las principales conclusiones del estudio. $\mathrm{Y}$ en la última se presentan las referencias bibliográficas utilizadas.

\section{DISEÑO METODOLÓGICO}

$\mathbf{P}$ ara realizar el proceso de aplicación de procesos asociados al plan de gestión de costos, se ha utilizado el proyecto "Construcción y montaje cubierta hornos, tolva san Carlos" de la empresa E01, cuyo contenido será descrito en detalle en el siguiente apartado.

En la fase exploratoria del estudio se ha realizado un diagnóstico respecto a la situación actual de los procedimientos que se realizan cuando se inicia un nuevo proyecto. Posteriormente, se han diseñado y documentado los procesos de iniciación, planeación, ejecución, control y monitoreo, y cierre del proyecto mencionado, específicamente en el área del conocimiento de gestión de costos.
Por último, aplicando la investigación-acción, se han implementado las adaptaciones mencionadas en el proyecto "Construcción y montaje cubierta hornos, tolva San Carlos", verificando los resultados de la aplicación de las técnicas y herramientas empleadas.

El alcance del trabajo de investigación se enfoca sobre la verificación de que la propuesta del estándar del PMI puede ser aplicable en el entorno colombiano. Las limitaciones se evidenciaron en la recolección y ajuste de la información relacionada con la aplicación.

A continuación se presenta un esquema del diseño metodológico establecido para cada una de las etapas del trabajo de investigación (tabla 1). 
Tabla 1. Diseño metodológico.

\begin{tabular}{|c|c|c|}
\hline Actividad & Técnicas y herramientas & Entregable \\
\hline $\begin{array}{l}\text { Diagnóstico respecto a la situación actual del } \\
\text { manejo de los costos del proyecto. }\end{array}$ & $\begin{array}{l}\text { Recopilación de la información respectiva del } \\
\text { proyecto (Análisis de Precios Unitarios APU, } \\
\text { presupuesto, propuesta, cronograma, recursos } \\
\text { rumanos RRHH, etc.). }\end{array}$ & Diagnóstico de la gestión del proyecto. \\
\hline \multirow{10}{*}{$\begin{array}{l}\text { Diseñar y documentar los procesos de } \\
\text { iniciación, planeación, ejecución, control } \\
\text { y monitoreo, y cierre del proyecto "Cons- } \\
\text { trucción y montaje cubierta hornos, tolva } \\
\text { San Carlos", en las áreas del conocimiento } \\
\text { de gestión de costos. }\end{array}$} & \multicolumn{2}{|c|}{ Proceso de iniciación } \\
\hline & $\begin{array}{l}\text { Juicio de expertos, documentación legal del } \\
\text { proyecto. }\end{array}$ & $\begin{array}{l}\text { Chárter del proyecto e identificación de } \\
\text { Stakeholders. }\end{array}$ \\
\hline & \multicolumn{2}{|c|}{ Procesos de planeación } \\
\hline & $\begin{array}{l}\text { Identificación y definición de las estructuras } \\
\text { del área de conocimiento para su respectiva } \\
\text { planeación. }\end{array}$ & Plan para la dirección del proyecto. \\
\hline & \multicolumn{2}{|c|}{ Procesos de ejecución } \\
\hline & $\begin{array}{l}\text { Desarrollo del plan de gestión de costos. } \\
\text { Trazabilidad de cada una de las actividades del } \\
\text { proyecto. } \\
\text { Sistema de información para la dirección del } \\
\text { proyecto. }\end{array}$ & $\begin{array}{l}\text { Indicadores sobre el desempeño del trabajo. } \\
\text { Solicitudes de cambio. } \\
\text { Actualizaciones al plan para la dirección } \\
\text { del proyecto. } \\
\text { Actualizaciones a documentos. }\end{array}$ \\
\hline & \multicolumn{2}{|c|}{ Procesos de control y monitoreo } \\
\hline & Desarrollo del control de costos. & Control de costos. \\
\hline & \multicolumn{2}{|c|}{ Procesos de cierre } \\
\hline & $\begin{array}{l}\text { Entregables aceptados. } \\
\text { Información histórica y base de conocimientos } \\
\text { de lecciones aprendidas. }\end{array}$ & $\begin{array}{l}\text { Transferencia del producto, servicio o re- } \\
\text { sultado final. } \\
\text { Actualizaciones a los activos de los pro- } \\
\text { cesos de la organización. }\end{array}$ \\
\hline $\begin{array}{l}\text { Implementar el plan de gestión para el } \\
\text { proyecto "Construcción y montaje cubierta } \\
\text { hornos, tolva San Carlos, en el área del } \\
\text { conocimiento de gestión de costos. }\end{array}$ & $\begin{array}{l}\text { Herramientas informáticas, documentación y } \\
\text { entregables diseñados en las etapas anteriores. }\end{array}$ & $\begin{array}{l}\text { Plan de gestión en el área de gestión de } \\
\text { costos. }\end{array}$ \\
\hline
\end{tabular}

Fuente. Elaboración propia. 


\section{DESCRIPCIÓN DEL CONTEXTO}

$\mathbf{L}$ a aplicación se desarrolla en la empresa E01. Sus oficinas administrativas se encuentran ubicadas en la ciudad de Duitama (Boyacá, Colombia) y el taller de fabricaciones se encuentra en Tunja (Boyacá, Colombia). La empresa E01 está especializada en el diseño y fabricación de estructuras metálicas, montajes mecánicos y servicios de mantenimiento para el sector minero e industrial.

El proyecto analizado se realizó para la planta San Carlos del cliente E02, ubicada en el municipio de Guachetá,

Tabla 2. Estructuras y bandas transportadoras diseño de la empresa E01.

\begin{tabular}{|c|c|c|}
\hline 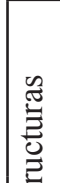 & $\begin{array}{l}\text { Tolvas de } \\
\text { mezcla - } \\
\text { capacidad } 35 \\
\text { toneladas } \\
\end{array}$ & $\begin{array}{l}\text { Tres tolvas de capacidad de } 35 \text { toneladas } \\
\text { (estructura, tolvas, cerramiento y cubierta) } \\
\text { para la dosificación y mezcla del carbón. }\end{array}$ \\
\hline 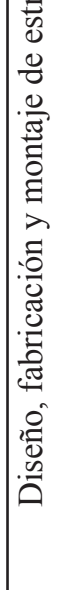 & 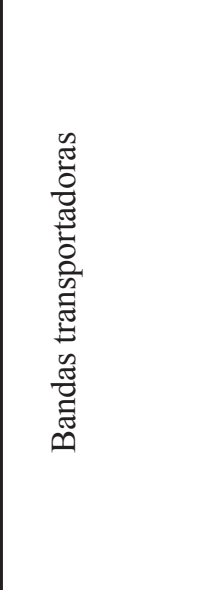 & $\begin{array}{l}\text { Tres bandas transportadoras de } 36 \\
\text { pulgadas de ancho y con una capacidad de } \\
200 \text { toneladas/hora cada una. La primera, } \\
\text { de } 56 \text { metros de longitud, que conduce la } \\
\text { mezcla desde las tolvas de } 35 \text { toneladas } \\
\text { a la torre de trituración (Banda B1). La } \\
\text { segunda (Banda B2), de } 232 \text { metros, } \\
\text { que conduce la mezcla triturada hacia } \\
\text { la torre de transferencia. Y una tercera, } \\
\text { de } 56 \text { metros (Banda B3), que va desde } \\
\text { la torre de transferencia hasta la tolva de } \\
\text { alimentación de los hornos y tiene una } \\
\text { capacidad de almacenamiento de } 500 \\
\text { toneladas (Tolva } 500 \text { toneladas). }\end{array}$ \\
\hline
\end{tabular}

Fuente. Elaboración propia basada en los diseños.
Cundinamarca. La empresa E01 realizó el diseño, la fabricación y el montaje de las estructuras para el sistema de alimentación de carbón molido para hornos de solera.

Se resumen las estructuras y bandas que fueron diseñadas y calculadas por la empresa E01 (tabla 2) se presentan las estructuras cuyos diseños fueron suministrados por la empresa E02 (tabla 3).

Tabla 3. Estructuras diseño de la empresa E02.

\begin{tabular}{|c|c|c|c|}
\hline $\begin{array}{c}\text { Torre } \\
\text { Molinos o } \\
\text { torre de } \\
\text { Trituración }\end{array}$ & $\begin{array}{l}\text { Estructura torre Molinos o torre de Trituración } \\
\text { que soporta dos molinos de martillos que se } \\
\text { alimenta por la banda 1 B1 y descarga el carbón } \\
\text { molido por medio de un shute bifurcado a la } \\
\text { banda 2 B2. Lleva un cerramiento perimetral } \\
\text { para evitar la contaminación producida por los } \\
\text { finos a la atmósfera. }\end{array}$ \\
\hline $\begin{array}{c}\text { Torre de } \\
\text { transfe- } \\
\text { rencia }\end{array}$ & $\begin{array}{l}\text { Es una estructura cuya finalidad es hacer un } \\
\text { cambio de dirección de 90 entre las bandas } \\
\text { B2 y B3 y unir la torre Molinos con la tolva de } \\
\text { almacenamiento. }\end{array}$ \\
\hline $\begin{array}{c}\text { Tolva de } \\
\text { alimenta- } \\
\text { ción- } \\
\text { capacidad } \\
\text { 500 ton. }\end{array}$ & $\begin{array}{l}\text { Una estructura de soporte de la tolva de } \\
\text { alimentación, de capacidad 500 toneladas, con } \\
\text { dos plataformas de mantenimiento y la tolva de } \\
\text { alimentación de la máquina cargadora de los } \\
\text { hornos con su respectiva cubierta. }\end{array}$ \\
\hline \multirow{2}{*}{$\begin{array}{c}\text { Estructura } \\
\text { cubierta } \\
\text { hornos }\end{array}$} & $\begin{array}{l}\text { Estructura de cuatro módulos de cubierta para } \\
\text { hornos de solera ubicados dos a cada lado de } \\
\text { la tolva de 500 toneladas. Cada módulo está } \\
\text { compuesto por nueve pórticos (1 pórtico = } 2 \\
\text { columnas y 1 cercha) cuya finalidad es cubrir } \\
\text { los hornos y dar soporte a la viga carrilera del } \\
\text { riel por donde se desplaza la máquina llenadora. }\end{array}$ \\
\cline { 2 - 3 }
\end{tabular}

Fuente. Elaboración propia basada en diseños de la empresa E02. 


\section{APLICACIÓN Y ANÁLISIS dE RESULTADOS}

E n este apartado se describe el proceso que se llevó a cabo para la aplicación del plan de gestión de costos en la empresa. Así mismo, se presentan todas las etapas e instrumentos aplicados, además de los resultados obtenidos consecuencia de dicha aplicación.

Figura 1. Mapa de procesos empresa E01.
El proceso de nuevos proyectos comienza cada vez que una cotización es aprobada para iniciar un nuevo proyecto en la Empresa E01. Se debe crear un centro de costos para llevar el seguimiento del mismo en términos de contabilidad y de los procedimientos internos de la empresa de acuerdo con el sistema de gestión que aplican. Se puede apreciar el mapa de procesos de la empersa (figura 1).

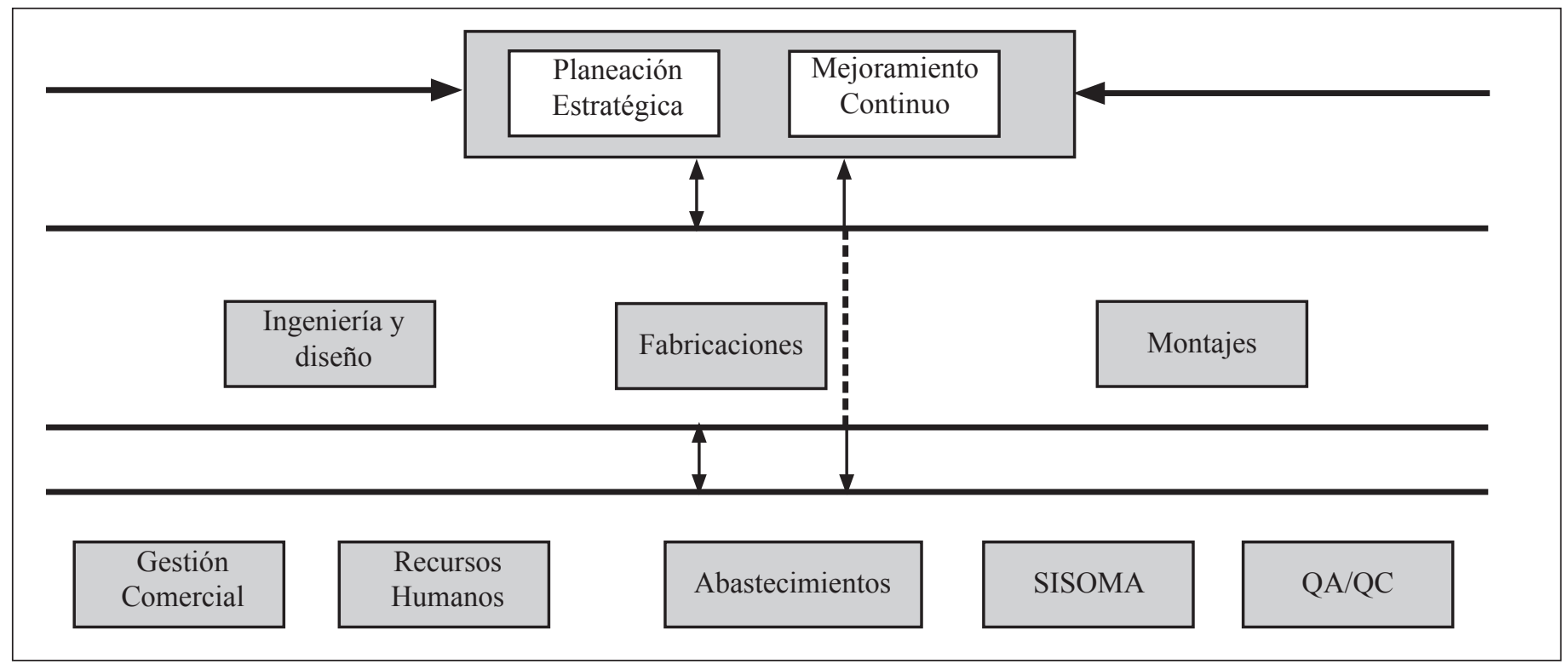

Fuente. Empresa E01.

El centro de costos se nombra con las iniciales ICM y un número consecutivo asignado al proyecto específico, en este caso es 153. Por lo tanto, en el presente documento se referirá a ICM 153, como nombre y centro de costos del proyecto.

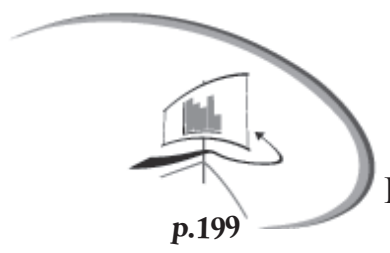

El primer proceso aplicado a partir del estándar PMI es la estimación de costos, para el cual se realiza un Análisis de Precios Unitarios (APU), un presupuesto y una propuesta, según los planos de diseño del cliente considerando el peso en kilogramos de las estructuras que componen el proyecto.

El APU asigna los precios unitarios a cada una de las etapas del proyecto en cuanto a las necesidades de equipos, materiales, transportes y mano de obra. El presupuesto contiene una descripción más detallada de los componentes de cada una de las etapas del proyecto, que es precisamente la Estructura Detallada de Trabajo (EDT), al igual que la cantidad, la unidad (en kilogramos, metros lineales, metros cuadrados, unidades), su valor unitario y su valor total.

En la estimación final de los costos se presenta el presupuesto general del proyecto para formalizar una negociación con el cliente, en donde se llega a un acuerdo en cuanto al precio a cobrar por $\mathrm{Kg}$ de estructura fabricada y montada. Para el costo de las bandas se consideraron solamente precios ya establecidos de venta al cliente, de acuerdo con las cantidades previamente calculadas.

El siguiente proceso del estándar, denominado "determinar el presupuesto", se aplicó sumando los costos estimados de paquetes de trabajo para establecer la línea base de costo 
autorizada. Para determinar el presupuesto se realizan consideraciones bajo los supuestos de precios manejados en proyectos anteriores con el cliente y según cotizaciones vigentes para la fecha de la firma del contrato. Las salidas a considerar de este proceso, según la aplicación hecha a partir del estándar del PMI, son la Línea base del desempeño de costos y los Requisitos de financiamiento del proyecto. Estos se suplieron de acuerdo con los anticipos del cliente según lo acordado en la firma del contrato y en los cortes de obra.

Para el proceso de "control de costos", siguiendo las directrices del PMI, las técnicas y herramientas empleadas se basaron fundamentalmente en el seguimiento a cada uno de los elementos del costo, de acuerdo con el APU de las estructuras.

Se consideraron entonces, datos concernientes a la nómina, a las requisiciones de materiales en fabricación y en montaje, y a las cuentas generadas para este centro de costos en contabilidad. Al igual que la nómina administrativa y los gastos que la obra asumió dentro de la empresa.

Para la asignación de los gastos y de las cuentas a prorratear se empleó el direccionador del costo de mano de obra directa (la sumatoria de fabricación y montaje), con respecto al total de la mano de obra utilizada. La asignación de los porcentajes para cada mes para el proyecto ICM 153, se asignó según la figura 2.

Figura 2. Horas de mano de obra empresa E01 para el prorrateo del centro de costos ICM 153.

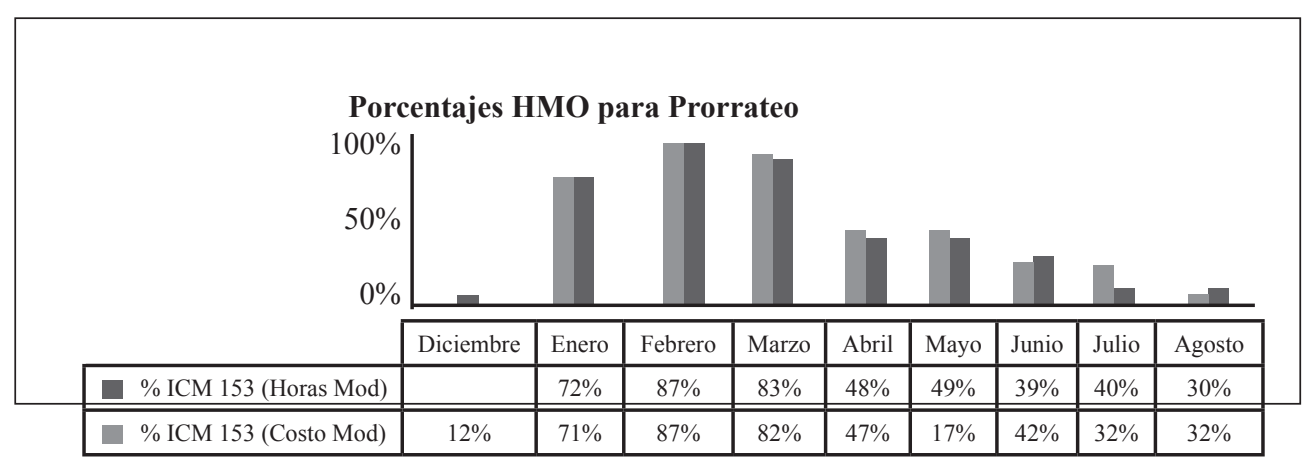

Fuente. Elaboración propia.

Adicionalmente, para obtener el costo unitario por Kg para los ítems requeridos, se asignó el cociente entre el valor correspondiente por el prorrateo y la cantidad de kilogramos fabricados mensualmente para el proyecto, de acuerdo con la información de los avances de obra del mismo (figura 3).

Figura 3. Producción enero-agosto 2012. ICM 153

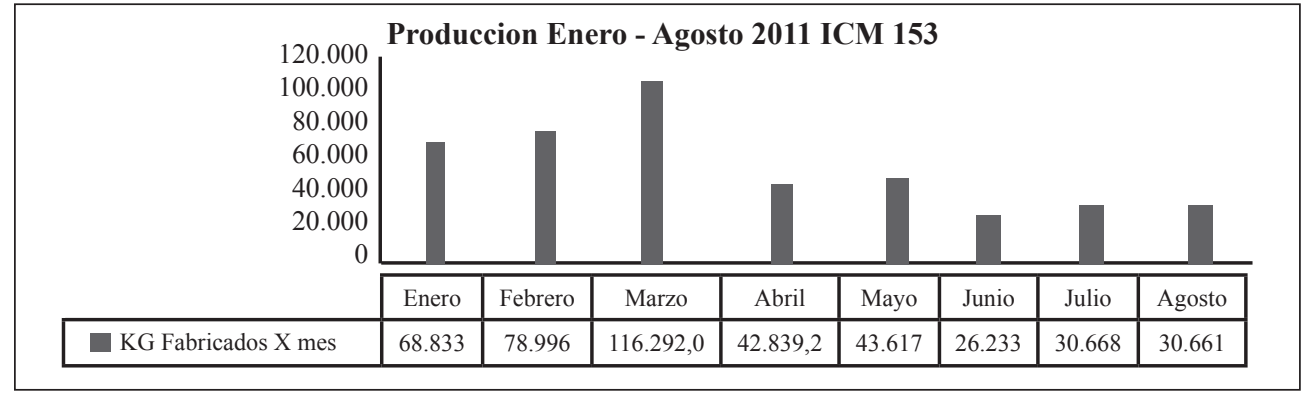

Fuente. Elaboración propia. 
Para los elementos del costo de materiales y mano de obra se consideraron solo los costos asumidos directamente por el proyecto ICM 153. En el caso de los ítems de equipo, transporte y administración, se hizo un prorrateo según las condiciones descritas anteriormente. Para el análisis del factor prestacional y los imprevistos se generaron cálculos independientes.

El APU contempla el análisis para dos capítulos: fabricación y montaje en el que cada uno consta de un desglose para: equipo, materiales, transporte y mano de obra. Otros aspectos considerados en el APU son: factor prestacional y AIU: Administración, Imprevistos y Utilidades. La salida representativa del control de costos es la comparación entre el APU inicial y el APU real. A continuación se explica la forma como se analizó cada ítem del APU tanto para fabricación como para montaje.

\subsection{Análisis de precios unitarios reales 4.1.1 Capítulo: fabricaciones}

\subsubsection{Equipo}

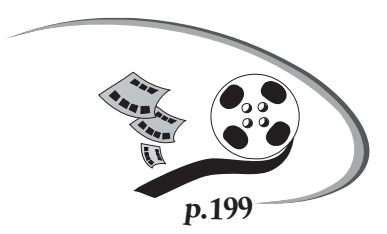

Para este ítem se considera lo correspondiente a energía y servicios, instalaciones, equipos y herramientas.

Los tres se cobran según una unidad global, una tarifa por hora y un rendimiento de una unidad.

Energía y servicios: corresponde a los costos generados por concepto de energía eléctrica, servicio de agua, Internet y teléfono. Los dos primeros sólo incluyen la planta de fabricación, mientras que los dos últimos se consideraron para toda la empresa, pues encierran todos los procesos que intervienen en el proyecto y que son necesarios para el desarrollo del mismo. De acuerdo con esto, se describen los resultados obtenidos del análisis de cada uno.

Energía eléctrica: se utilizaron los datos que se presentan en la tabla 4. El costo unitario resulta del promedio del valor de todos los meses, y es de $\$ 51,57 / \mathrm{Kg}$.
Tabla 4. Costo energía eléctrica ICM 153.

\begin{tabular}{|c|c|r|}
\hline \multicolumn{2}{|c|}{ COSTO ENERGÍA ELÉCTRICA ICM 153 } \\
\hline MES & $\begin{array}{c}\text { VALOR TOTAL } \\
\text { ICM 153 }\end{array}$ & $\begin{array}{c}\text { COSTO } \\
\text { UNITARIO }\end{array}$ \\
\hline Enero & $\$ 2.718 .095,25$ & $\$ 39,49$ \\
\hline Febrero & $\$ 4.066 .621,38$ & $\$ 51,48$ \\
\hline Marzo & $\$ 3.496 .702,03$ & $\$ 30,07$ \\
\hline Abril & $\$ 1.731 .402,86$ & $\$ 40,42$ \\
\hline Mayo & $\$ 2.590 .004,55$ & $\$ 59,38$ \\
\hline Junio & $\$ 2.167 .413,17$ & $\$ 82,62$ \\
\hline Julio & $\$ 1.357 .392,12$ & $\$ 44,26$ \\
\hline Agosto & $\$ 1.988 .553,94$ & $\$ 64,86$ \\
\hline $\begin{array}{l}\text { COSTO } \\
\text { TOTAL }\end{array}$ & $\$ 20.454 .743,25$ & \\
\hline $\begin{array}{l}\text { COSTO } \\
\text { UNITARIO }\end{array}$ & & \multicolumn{2}{|c}{$\$$} \\
\hline
\end{tabular}

Fuente. Elaboración propia.

Servicio de agua: realizando cálculos similares al presentado para el costo de energía, obtuvo un costo unitario de $\$ 0,06 / \mathrm{Kg}$.

Internet: se utilizó la misma metodología de cálculo y se obtuvo que el costo unitario de servicio de Internet para el proyecto es de $\$ 3,50 / \mathrm{Kg}$.

Teléfono: para obtener el costo de teléfono se consideraron las líneas utilizadas en fabricación, montaje, abastecimientos y administración en Telefónica, obteniendo un costo unitario de $\$ 14,39 / \mathrm{Kg}$.

El costo unitario por concepto de energía y servicios fue de $\$ 69,52 / \mathrm{Kg}$, calculado mediante la sumatoria de cada uno de los precios unitarios. Realizando la comparación del APU presupuestado con el APU real, se encuentró una diferencia de $\$ 30,48$, por lo que se puede afirmar que el costo estaría dentro del rango positivo (el real es menor al presupuestado) de control del costo.

\subsubsection{Instalaciones}

El valor a analizar corresponde a los costos atribuidos únicamente a la planta de fabricación (tabla 5). 
Tabla 5. Costo de instalaciones ICM 153.

\begin{tabular}{|l|r|}
\hline \multicolumn{2}{|c|}{ COSTO DE INSTALACIONES ICM 153 } \\
\hline \multicolumn{1}{|c|}{ CONCEPTOS } & TOTAL \\
\hline Arriendo taller & $\$ 13.603 .167,50$ \\
\hline Aseo y vigilancia taller & $\$ 621.926,37$ \\
\hline Reparaciones locativas & $\$ 7.338 .868,30$ \\
\hline COSTO TOTAL & $\$ 21.563 .962,18$ \\
\hline COSTO UNITARIO & $\$$ \\
\hline
\end{tabular}

Fuente. Elaboración propia.

En cuanto a este ítem en el APU presupuestado se cobró $\$ 100$, con lo que se obtiene una diferencia de \$53 del APU real.

\subsubsection{Mano de obra}

Se tomó como referencia el costo asignado a cada operario según el cargo y de acuerdo con el documento Suministro Personal a Terceros, procedente de estudios anteriores en la empresa.

Para el proceso de armado se necesitaron 9.611 horas -hombre, de acuerdo con las horas de mano de obra por clase de tarea y teniendo en cuenta que el total de las toneladas fabricadas son 364, que la producción promedio de la obra es de 1,5 ton/día, dato obtenido del Resumen Producción ICM-153, y que cada día se laboran ocho horas, por tanto la cantidad de hombres necesarios está dada por la siguiente fórmula:

$$
\begin{gathered}
\frac{9611 \text { horas-hombre }}{364 \text { ton }} * 1,5 \frac{\text { ton }}{\text { día }} * \frac{1 \text { día }}{8 \text { hras }}=4,95 \text { hombres } \\
\approx 5 \text { hombres para Armado }
\end{gathered}
$$

De la misma manera se analizó por cada tarea y cargo correspondiente a la mano de obra considerada en el APU.

Un caso especial surge en las horas para obtener la cantidad de ayudantes técnicos y ayudantes. Para estos cargos se relacionaron las horas de corte, preparación y taladro; luego, se aplicó el porcentaje según el cargo, para tener las horas totales de cada uno. La planta de fabricación cuenta con nueve ayudantes técnicos y 20 ayudantes, razón por la cual el $31 \%$ de las horas serán aplicadas a los primeros y el $69 \%$, a los segundos. Así, la cantidad de ayudantes técnicos necesarios son:

$$
\begin{gathered}
\frac{6947,91 \text { hras-hombre }}{\text { (364 ton })} * 1,5 \frac{\text { ton }}{\text { día }} * \frac{1 \text { día }}{8 \text { hras }}=3,3 \text { hombres } \\
\approx 3 \text { Ayudantes Técnicos }
\end{gathered}
$$

El resumen de estos cálculos se muestran en la tabla 6.

Tabla 6. Cantidad mano de obra para fabricación.

\begin{tabular}{|l|r|r|r|c|}
\hline \multicolumn{1}{|c|}{ CARGO } & $\begin{array}{c}\text { HRS- } \\
\text { HOMBRE }\end{array}$ & $\begin{array}{c}\text { H-H/ } \\
\text { TON }\end{array}$ & $\begin{array}{c}\text { H-H/ } \\
\text { DIA }\end{array}$ & $\begin{array}{c}\text { HOM- } \\
\text { BRES }\end{array}$ \\
\hline Ayudantes I & 12786,21 & 35,1 & 52,7 & 7 \\
\hline Ayudante técnico I & 6497,91 & 17,9 & 26,8 & 3 \\
\hline Armador I & 9611 & 26,40 & 39,61 & 5 \\
\hline Pintura & 4872 & 13,4 & 20,1 & 3 \\
\hline Soldador & 5427 & 14,91 & 22,36 & 3 \\
\hline
\end{tabular}

Fuente. Elaboración propia.

\subsubsection{Herramientas}

Dentro de las consideraciones para equipo y herramientas se tienen en cuenta tanto los equipos más representativos dentro de los activos fijos de la empresa, como las herramientas menores que requiere cada operario. Finalmente, de acuerdo

\begin{tabular}{|c|c|c|c|}
\hline \multicolumn{4}{|c|}{ HERRAMIENTAS - FABRICACIÓN } \\
\hline OPERARIO & $\begin{array}{c}\text { CANTIDAD } \\
\text { DE } \\
\text { OPERARIOS }\end{array}$ & $\begin{array}{c}\text { COSTO } \\
\text { UNID. }\end{array}$ & $\begin{array}{l}\text { COSTO } \\
\text { TOTAL }\end{array}$ \\
\hline Supervisor & 1 & & $\$$ \\
\hline Soldador I & 3 & $\$ 10.000,00$ & $\$ 28.000,00$ \\
\hline Armador I & 5 & $\$ 10.000,00$ & $\$ 49.000,00$ \\
\hline $\begin{array}{l}\text { Ayudante } \\
\text { técnico I }\end{array}$ & 3 & $\$ 6.500,00$ & $\$ 21.450,00$ \\
\hline Ayudante & 7 & $\$ 5.000,00$ & $\$ 33.000,00$ \\
\hline Pintor & 3 & $\$ 5.000,00$ & $\$ 12.500,00$ \\
\hline \multicolumn{4}{|l|}{ Almacenista } \\
\hline \multirow[t]{2}{*}{ TOTAL } & 21 & & $\$ 143.950,00$ \\
\hline & \multicolumn{2}{|c|}{ COSTO UNITARIO } & 95,97 \\
\hline
\end{tabular}
con la cantidad de personal necesario para fabricación (tabla 6), se asignó el valor correspondiente (tabla 7).

Tabla 7. Costo herramientas para fabricación.

Fuente. Elaboración propia.

Para el APU presupuestado se consideró un costo de \$200, y para el APU real, uno de $\$ 98,63$ lo que indica un margen de diferencia positiva significante. 
En general, para los ítems que integran la parte correspondiente a 'equipo', se consideraron montos superiores y el costo real contribuye a una ganancia positiva para el proyecto de casi el $50 \%$.

\subsubsection{Materiales}

Este ítem se considera representativo dentro del APU, ya que el cliente E02 suministró todo el material directo para las estructuras, por lo tanto a la Empresa E01 le corresponde sólo la administración de todos los materiales. Teniendo en cuenta lo anterior, para el análisis se consideró sólo lo correspondiente a consumibles y oxicorte, soldaduras, anticorrosivo gris corriente y esmalte alquílico. Los datos utilizados se obtuvieron de las requisiciones de fabricaciones. Se describe a continuación el procedimiento utilizado para cada uno.

Consumibles y oxicorte. Del comparativo de las requisiciones y órdenes de compra de fabricaciones, se consideraron los materiales clasificados como consumibles en el kárdex general. El valor total de estos en fabricación para el proyecto, asciende a $\$ 19.206 .550,28$. Por lo tanto, el costo unitario es de $\$ 52,63$, considerando una fabricación total de $364.905 \mathrm{Kg}$.

Se observa que en el APU inicial se presupuesta un valor unitario de \$200. Si se analiza únicamente este ítem, se concluye que el valor cobrado globalmente está por encima del real en un $73 \%$.

Soldaduras. Se identificó un consumo de soldadura eléctrica de $2340 \mathrm{Kg}$, de soldadura mig de $75 \mathrm{Kg}$, de soldadura de arco sumergido de $300 \mathrm{Kg}$ y de soldadura tubular de $1240 \mathrm{Kg}$. Aun cuando el rendimiento de las dos es diferente, se debe considerar que el precio de la soldadura tubular es de $\$ 10440 / \mathrm{Kg}$ y el de la soldadura eléctrica de $\$ 6650 / \mathrm{Kg}$.

En general, la cantidad de soldadura consumida por fabricaciones fue de $3955 \mathrm{Kg}$ a un costo promedio de \$7291,31/ $\mathrm{Kg}$, diferente en $\$ 99,31$ de $\$ 7192 / \mathrm{Kg}$, que fue el valor con el que se presupuestó. En cuanto al rendimiento se observó que para las 364 toneladas es fue del 1,1\% y se había estimado el 1,5\%.

Anticorrosivo corriente gris. El rendimiento presupuestado fue del $0,29 \%$ y el rendimiento real de $0,17 \%$. De tal manera que su costo unitario fue de $\$ 36 / \mathrm{Kg}$. Se destaca una disminución considerable en este ítem.

Esmalte alquílico. El costo real para el esmalte, incluyendo el del varsol, es de $\$ 39534,04 / \mathrm{Gl}$, y su rendimiento es el mismo que para el anticorrosivo. El costo unitario calcula un valor de $\$ 66 / \mathrm{Kg}$.

\subsubsection{Transporte}

Según el APU inicial, no se suma costo para el transporte en el capítulo de fabricaciones, es decir, el costo de transporte, tanto de material como de estructuras, se lleva directamente al capítulo de montaje. Pero realmente estos dos ítems se llevan a cabo en fabricaciones.

Para obtener el costo unitario, se dividió el costo total causado por el proyecto en el total de los kilogramos transportados, de lo cual resultó un valor unitario de $\$ 107,63 / \mathrm{Kg}$. En conclusión, en el APU inicial se contemplaronó \$200 para transporte, $\$ 50$ para materiales y $\$ 150$ para estructura. En contraste con lo presupuestado los valores reales se muestran inferiores y tienen un margen de diferencia de casi el $52 \%$ y el $59 \%$, respectivamente.

\subsubsection{Mano de obra}

Este ítem incluye el costo por concepto de remuneración para los siguientes cargos: dibujante de taller, ingeniero residente, supervisor de fabricación, supervisor de seguridad, armador I, ayudante técnico I, ayudante I, soldador I y pintor. La unidad a presupuestar es en días, el jornal total corresponde al salario de un día de trabajo según el cargo, el rendimiento está dado de acuerdo con el número de trabajadores por el total de días a utilizar en la fabricación de las 364 toneladas.

Se tuvieron en cuenta las horas correspondientes a trabajo con estructura pesada, debido a que el APU está directamente relacionado con esta clase de tarea. Se contaron los días que se pagaron y se dividió en la cantidad de $\mathrm{Kg}$ fabricados para obtener el rendimiento. Entonces el valor parcial (Jornal Total * Rendimiento), sólo es superior para el pintor y para el supervisor de fabricación, ya que alcanzan un valor de $\$ 19 / \mathrm{Kg}$ y de $\$ 8 / \mathrm{Kg}$, mientras que en el APU presupuestado se cobró en $\$ 17 / \mathrm{Kg}$ y $\$ 3 / \mathrm{Kg}$ respectivamente. 


\subsubsection{Análisis general APU fabricaciones}

Según los costos presu-puestados y realmente gastados dentro de cada ítem, se asigna un porcentaje que refleja el cambio del APU inicial al APU real (tabla 8). Se encuentra la presencia del costo de transporte dentro del APU de fabricación, un aumento del $11 \%$ en la mano de obra y una disminución en los restantes ítems generales. Los porcentajes se dan de acuerdo con la variación de la tarifa por hora, el valor unitario, la tarifa y el jornal total, respectivamente.
Las columnas 'Inicial' y 'Final' hacen referencia sólo al cambio del costo, mientras que las columnas ' $\%$ Inicial' y ' $\%$ Final' indican el porcentaje asignado dentro del APU para cada ítem con respecto al total del mismo (tabla 8). Por lo tanto, se reflejarán disminuciones de costos, acompañados por porcentajes de variación aumentados, como el caso de mano de obra, pues aunque se muestra un descenso en la mayoría de los cargos, sí hubo una mayor utilización de recursos. Se concluye que aún con el aumento del porcentaje de la mano de obra, el costo del $\mathrm{Kg}$ de fabricación se encuentra dentro del valor cobrado para el presupuesto.

Tabla 8. Análisis general APU fabricaciones.

\begin{tabular}{|c|c|c|c|c|c|c|}
\hline I. EQUIPO & $\mathbf{U N}$ & Inicial & Real & $\%$ Inicial & $\%$ Real & Observaciones \\
\hline Energía y servicios & gbl & $100 \%$ & $69,52 \%$ & \multirow{3}{*}{$21 \%$} & \multirow{3}{*}{$16 \%$} & \\
\hline Instalaciones & $\mathrm{gbl}$ & $100 \%$ & $46,91 \%$ & & & \\
\hline Equipos y herramientas & $\mathrm{gbl}$ & $100 \%$ & $47,98 \%$ & & & \\
\hline II. MATERIALES & $\mathbf{U N}$ & Inicial & Real & $\%$ Inicial & $\%$ Real & Observaciones \\
\hline Consumibles y oxicorte & gbl & $100 \%$ & $26,31 \%$ & \multirow{4}{*}{$32 \%$} & \multirow{4}{*}{$18 \%$} & Disminuyó \\
\hline Soldaduras AWS 70XX, 60XX & $\mathrm{kg}$ & $100 \%$ & $101,4 \%$ & & & Disminuyó \\
\hline Anticorrosivo corriente gris & gl. & $100 \%$ & $50,6 \%$ & & & Disminuyó \\
\hline Esmalte alquílico & gl. & $100 \%$ & $65,54 \%$ & & & Disminuyó \\
\hline III. TRANSPORTE & $\mathbf{U N}$ & Inicial & Real & $\%$ Inicial & $\%$ Real & Observaciones \\
\hline Equipo y materiales & gbl & $0 \%$ & $100 \%$ & \multirow[t]{2}{*}{$0 \%$} & \multirow[t]{2}{*}{$8 \%$} & $\begin{array}{l}\text { Se consideró en el } \\
\text { capítulo de montaje }\end{array}$ \\
\hline Estructura & $\mathrm{gbl}$ & $0 \%$ & $100 \%$ & & & \\
\hline IV. MANO DE OBRA & $\mathbf{U N}$ & Inicial & Real & $\%$ Inicial & $\%$ Real & Observaciones \\
\hline Dibujante de taller & $\mathrm{dd}$ & $100 \%$ & $101,3 \%$ & \multirow{10}{*}{$47 \%$} & \multirow{10}{*}{$58 \%$} & $\begin{array}{l}\text { Cuatro dibujantes por } \\
\text { cinco meses (real) }\end{array}$ \\
\hline Ingeniero residente & $\mathrm{dd}$ & $100 \%$ & $81 \%$ & & & Coor. Fabricaciones \\
\hline Supervisor fabricación & dd & $100 \%$ & $91,1 \%$ & & & Disminuyó \\
\hline Supervisor de seguridad & $\mathrm{dd}$ & $100 \%$ & $39,6 \%$ & & & Costo inicial elevado. \\
\hline Armador I & $\mathrm{dd}$ & $100 \%$ & $82,3 \%$ & & & Disminuyó \\
\hline Ayudante técnico I1 & $\mathrm{dd}$ & $100 \%$ & $75,1 \%$ & & & Disminuyó \\
\hline Ayudante I1 & dd & $100 \%$ & $68,9 \%$ & & & Disminuyó \\
\hline Soldador I & dd & $100 \%$ & $69,5 \%$ & & & Disminuyó \\
\hline Pintor & $\mathrm{dd}$ & $100 \%$ & $68,97 \%$ & & & Disminuyó \\
\hline Factor prestacional & $\%$ & $100 \%$ & $99,8 \%$ & & & \\
\hline
\end{tabular}

Fuente. Elaboración propia. 


\subsubsection{Capítulo: montaje}

Se realizó un análisis similar aplicado para fabricación, pero con la diferencia en que la cantidad de personal requerido se obtuvo de los operarios que en promedio trabajaron en el montaje del proyecto; así mismo, se hizo una comparación igual que para el capítulo de Fabricaciones (tabla 9). Se encuentra un aumento del $40 \%$ en la mano de obra y una disminución en los restantes ítems generales. Sin embargo, con el aumento del porcentaje de la mano de obra, el costo del $\mathrm{Kg}$ de montaje se encuentra dentro del valor cobrado para el presupuesto.

Tabla 9. Análisis general APU montaje.

\begin{tabular}{|c|c|c|c|c|c|c|}
\hline I. EQUIPO & $\mathbf{U N}$ & Inicial & Real & $\%$ Inicial & $\%$ Real & Observaciones \\
\hline Equipos y herramientas & gbl & $100 \%$ & $44,8 \%$ & $42 \%$ & $22 \%$ & \\
\hline Grúa & $\mathrm{gbl}$ & $100 \%$ & $42,7 \%$ & & & \\
\hline II. MATERIALES & $\mathbf{U N}$ & Inicial & Real & $\%$ Inicial & $\%$ Real & Observaciones \\
\hline Consumibles y oxicorte & $\mathrm{gbl}$ & $100 \%$ & $11,43 \%$ & $12 \%$ & $4 \%$ & \\
\hline $\begin{array}{l}\text { Soldaduras AWS 70XX, } \\
60 \mathrm{XX}\end{array}$ & $\mathrm{Kg}$ & & $100 \%$ & & & $\begin{array}{l}\text { No se había considerado } \\
\text { en el APU inicial. }\end{array}$ \\
\hline III. TRANSPORTE & $\mathbf{U N}$ & Inicial & Real & $\%$ Inicial & $\%$ Real & Observaciones \\
\hline Equipo y materiales & $\mathrm{gbl}$ & $100 \%$ & $0 \%$ & & & Se considera en el capí- \\
\hline Estructura & $\mathrm{gbl}$ & $100 \%$ & $0 \%$ & $12 \%$ & $0 \%$ & tulo de fabricación \\
\hline IV. MANO DE OBRA & $\mathbf{U N}$ & Inicial & Real & $\%$ Inicial & \% Real & Observaciones \\
\hline Ingeniero residente & $\mathrm{Dd}$ & $100 \%$ & $100,37 \%$ & \multirow{9}{*}{$34 \%$} & \multirow{9}{*}{$74 \%$} & $\begin{array}{l}\text { Se excluye de la nómina } \\
\text { administrativa }\end{array}$ \\
\hline Supervisor montaje & $\mathrm{Dd}$ & $100 \%$ & $94,5 \%$ & & & Disminuyó \\
\hline Supervisor de seguridad & Dd & $0 \%$ & $100 \%$ & & & $\begin{array}{l}\text { No estaba incluido en el } \\
\text { APU inicial }\end{array}$ \\
\hline Montador I & $\mathrm{Dd}$ & $100 \%$ & $103 \%$ & & & Aumentó \\
\hline Ayudante técnico. 1I & $\mathrm{Dd}$ & $100 \%$ & $121 \%$ & & & Aumentó \\
\hline Ayudante I1 & $\mathrm{Dd}$ & $100 \%$ & $120 \%$ & & & Aumentó \\
\hline Soldador I & $\mathrm{Dd}$ & $100 \%$ & $92,0 \%$ & & & Disminuyó \\
\hline Pintor & Dd & $100 \%$ & $116 \%$ & & & Aumentó \\
\hline Almacenista & $\mathrm{Dd}$ & $100 \%$ & $125 \%$ & & & Aumentó \\
\hline Factor prestacional & $\%$ & $100 \%$ & $99,8 \%$ & & & \\
\hline
\end{tabular}

Fuente. Elaboración propia.

\subsection{Análisis del factor administración, im- previstos y utilidades (AIU)}

Una vez analizados cada uno de los items del APU en los capítulos de Fabricación y de Montaje, se realizó en la parte final de la aplicación, el desglose de los componentes del factor Administración, Imprevistos y Utilidades (AIU).

\subsubsection{Administración}

Para los gastos asumidos por la administración de la empresa E01, se hizo un prorrateo de cada una de las cuentas de
Gastos Operacionales de Administración, de acuerdo con los porcentajes correspondientes del centro de costos ICM 153. Para este caso, no se determinó un valor por $\mathrm{Kg}$, ya que el gasto total y los costos directos del proyecto se obtienen del porcentaje de administración aplicado al proyecto de $\$$ 75.479.591, según la siguiente expresión:

$\%$ Administración $=\frac{\text { Gastos Totales ICM } 153}{\text { Costos Directos Totales ICM 153 }} * 100 \%$ 


\subsubsection{Determinación de los costos totales}

A continuación se describen los ítems que se tienen en cuenta dentro de cada uno de los elementos del costo para efectos de la determinación del total de los costos directos y los gastos consumidos por el proyecto (figura 4).

Figura 4. Elementos del costo
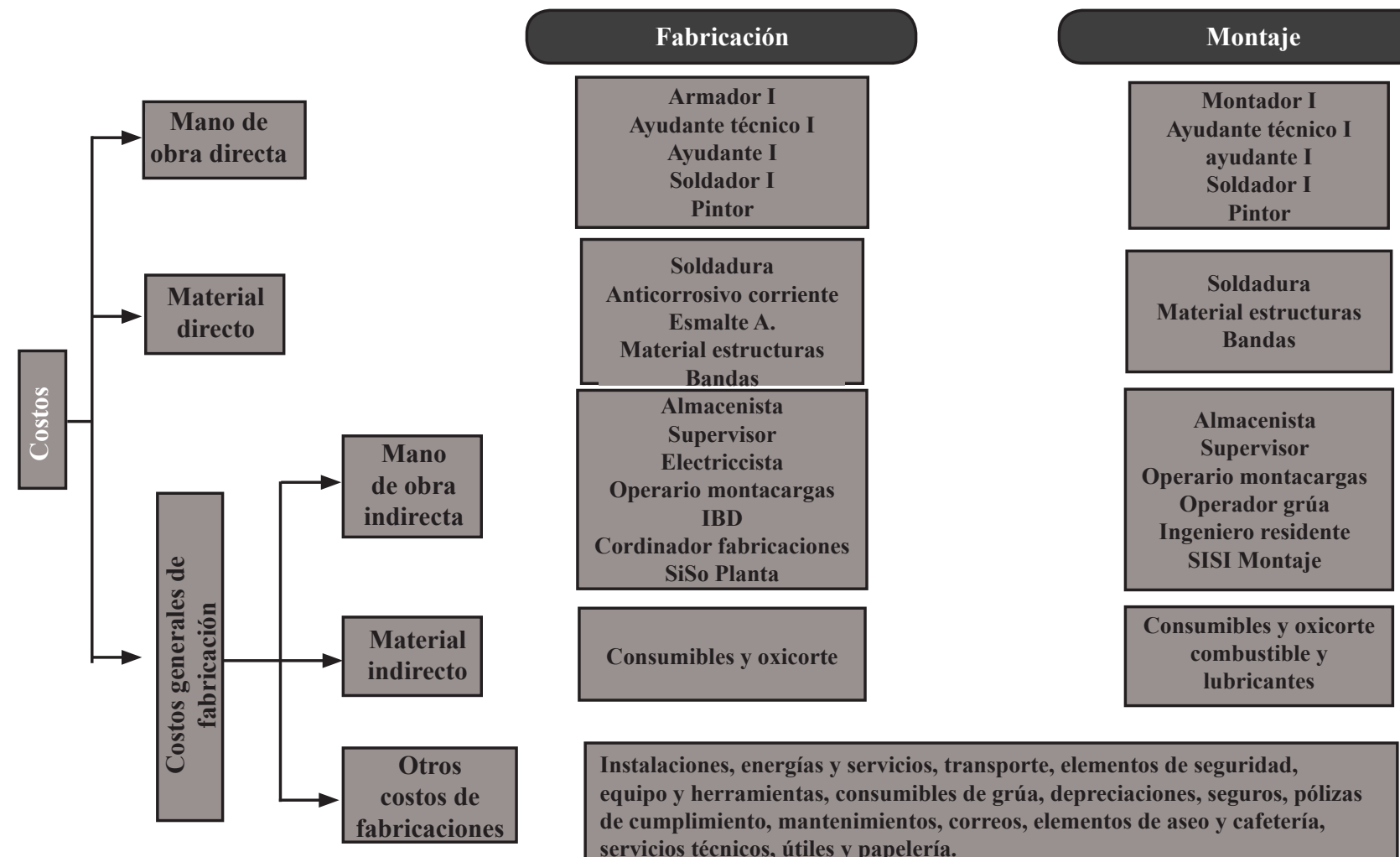

Consumibles y oxicorte

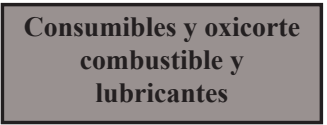

Instalaciones, energías y servicios, transporte, elementos de seguridad, equipo y herramientas, consumibles de grúa, depreciaciones, seguros, pólizas de cumplimiento, mantenimientos, correos, elementos de aseo y cafetería, servicios técnicos, útiles y papelería.

Fuente. Elaboración propia.

De acuerdo con los análisis realizados anteriormente se encuentra un porcentaje alto de administración, se hace la consideración de si la empresa E01 hubiera asumido el costo de materiales directos para estructuras y por el cual se asumiría un costo por $\mathrm{Kg}$ de $\$ 2650 / \mathrm{Kg}$ adicional. Así, los costos directos se incrementan y el porcentaje de administración arroja un valor de $17 \%$, lo cual es razonable de acuerdo con el APU para presupuestar.

\subsubsection{Imprevistos}

Dentro de los más representativos se encuentran los considerados de acuerdo con el tipo de imprevisto, la cantidad de operarios y el costo causado ( tabla 10). 
Tabla 10. Análisis detallado de imprevistos.

\begin{tabular}{|l|r|r|r|r|r|}
\hline \multicolumn{1}{|c|}{ RESUMEN } & HORAS & $\begin{array}{c}\text { \#OPERA- } \\
\text { TIVOS }\end{array}$ & $\begin{array}{c}\text { TIEMPO } \\
\text { TOTAL }\end{array}$ & \multicolumn{1}{c|}{$\begin{array}{c}\text { COSTO } \\
\text { TOTAL }\end{array}$} & $\begin{array}{c}\text { COSTO X } \\
\text { HORA }\end{array}$ \\
\hline Lluvia & 43,3 & 20 & 866,67 & $\$ 2.163 .741,6$ & $\$ 49.932,50$ \\
\hline Sin energía eléctrica & 4,6 & 20 & 91,67 & $\$ 332.190,83$ & $\$ 72.478,00$ \\
\hline Sin materiales & 13,0 & 11 & 143,00 & $\$ 454.692,88$ & $\$ 34.976,38$ \\
\hline Capacitaciones & 5,0 & 86 & 430,00 & $\$ 480.162,45$ & $\$ 96.032,49$ \\
\hline Exámenes médicos & 14,5 & 47 & 681,50 & $\$ 2.252 .450,3$ & $\$ 155.341,4$ \\
\hline $\begin{array}{l}\text { Incapacidad } \\
\text { montacargas }\end{array}$ & 3,0 & 4 & 12,00 & $\$ 92.939,25$ & $\$ 30.979,75$ \\
\hline $\begin{array}{l}\text { Aseo planta } \\
\text { fabricación }\end{array}$ & 2,0 & 64 & 128,00 & $\$ 413.486,75$ & $\$ 206.743,3$ \\
\hline Sin montacargas & 6,5 & 4 & 26,00 & $\$ 61.868,25$ & $\$ 9.518,19$ \\
\hline TOTAL & $\mathbf{9 1 , 9}$ & $\mathbf{2 5 6}$ & $\mathbf{2 . 3 7 8 , 8}$ & $\mathbf{\$ 6 . 2 5 1 . 5 3 2}$ & \\
\hline
\end{tabular}

Fuente. Elaboración propia.

Con respecto a los costos directos del proyecto, el porcentaje de imprevistos alcanzan un $0,54 \%$, dado por la siguiente expresión:

$$
\% \text { Imprevistos }=\begin{gathered}
\$ \text { Total Imprevistos } \\
\$ \text { Total Costos Directos }
\end{gathered}
$$

\subsubsection{Utilidades}

De acuerdo con los porcentajes de administración e imprevistos y según el escenario dos, que refleja lo realmente causado en el análisis de los costos totales, se llega a la conclusión que el porcentaje de utilidad es del 17\%.

Sin embargo, considerando que la administración del proyecto es del 17\% de acuerdo con la administración del material de estructuras, entonces la utilidad es del 34\%, una cifra positiva para la empresa que ha sido establecida con exactitud, al aplicar el procedimiento utilizado a partir del estándar del PMI.

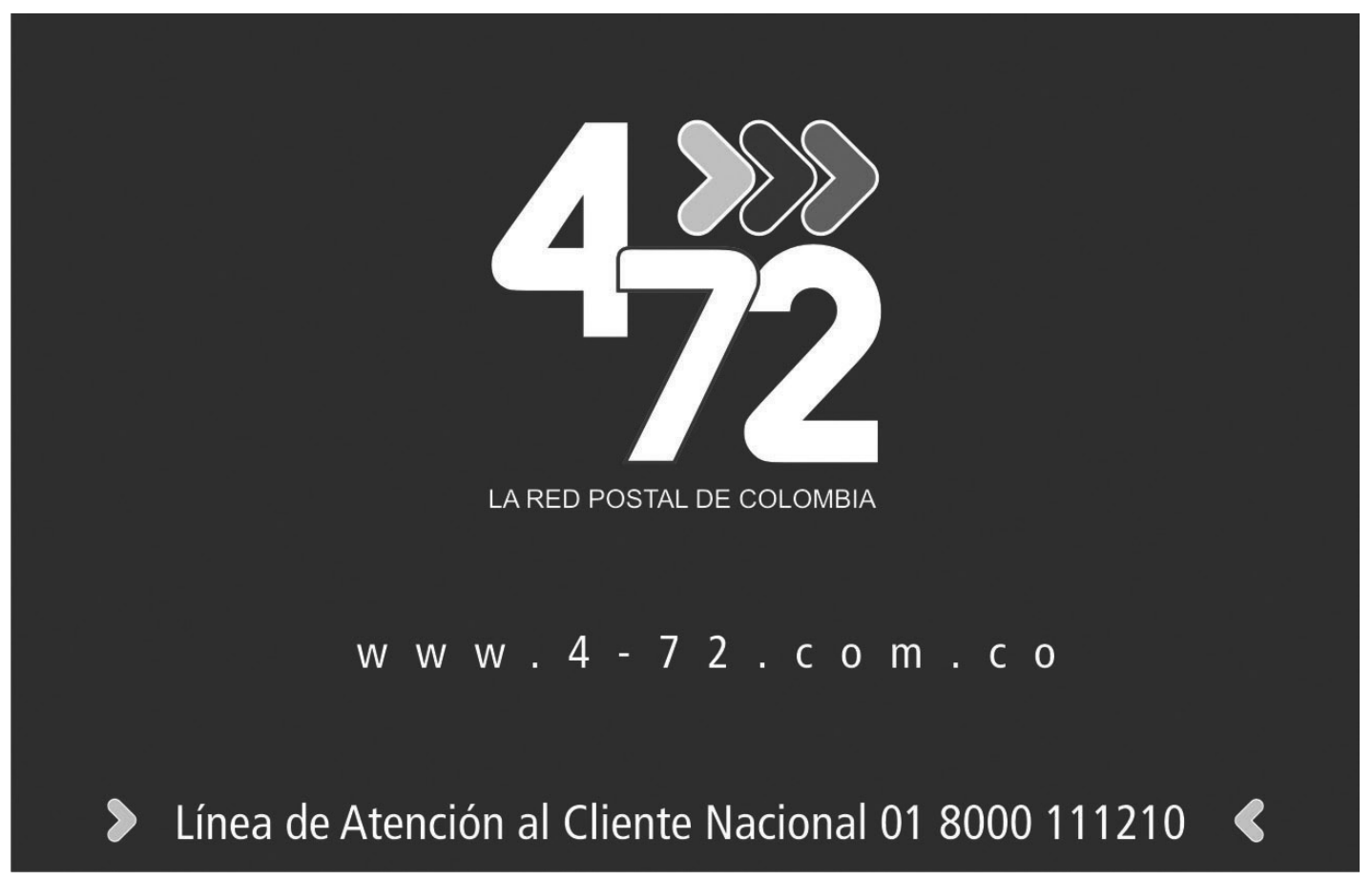




\section{CONCLUSIONES}

E n el presente artículo se ha documentado e ilustrado la aplicación de un plan de gestión de costos en proyectos de una empresa del sector minero-industrial de Colombia. Se ha encontrado un beneficio concreto en la aplicación de los procedimientos dispuestos por uno de los estándares internacionales de dirección de proyectos de mayor divulgación, como es el Project Management Nody of Knowledge (PMBOK) del Project Management Institute (PMI).

El diagnóstico de los procedimientos que se realizan en la empresa E01 para la ejecución de sus proyectos, señaló que las áreas de conocimiento del alcance, del tiempo y del costo, requerían del diseño y la implementación del Plan de Gestión de Proyectos, específicamente en los procesos de planeación y control.

Los valores reales obtenidos del análisis de costos de APU, pueden usarse para el control del presupuesto del proyecto y para asegurar que ninguno de los ítems de este sobrepasen su valor y se conserve la utilidad esperada.

En la mayoría de los ítems considerados en el APU real, se muestra una disminución del costo acompañado de un bajo rendimiento, comparado con el APU presupuestado, con lo que se obtiene un margen de utilidad positivo.

El análisis mensual de los ítems 'equipos', muestran un comportamiento del costo susceptible a la producción y al nivel de mano de obra utilizada, por lo que se sugiere controlar, mitigar o eliminar las causas de las actividades que sobrepasan el presupuesto inicial del proyecto.
En el ítem de 'materiales' se destaca que en el caso de los consumibles se consideraron como el doble de lo que realmente costaron, por tanto, se puede contemplar una disminución considerable para los nuevos APU que realice el departamento comercial de la empresa.

Se encontró una disminución en el costo de la mano de obra utilizada para la fabricación, acompañado de un mayor requerimiento de los recursos por lo que el costo unitario asumido por este ítem, aumentó considerablemente.

El costo de mano de obra de montaje no se consideró co-mo costos adicionales de alojamiento, alimentación y transporte, lo cual incidió en el aumento de este en un $40 \%$ más de lo presupuestado.

El porcentaje de administración superó el asignado en el presupuesto y se concluyó que aun cuando un proyecto sea grande o pequeño, económicamente hablando, el trabajo administrativo para la ejecución de este es el mismo. Por tanto, mientras más se extiendan los procesos para la finalización de un proyecto, mayor será el porcentaje utilizado por concepto de administración y en últimas, este terminará consumiendo el porcentaje de utilidad esperada.

El porcentaje asignado a la utilidad fue superior al presupuestado, por tanto se concluyó que, aun con las variaciones presentadas en el desarrollo del proyecto, se obtuvo una utilidad del $34 \%$ para la empresa. 


\section{REFERENCIAS}

Abbasi, G. Y. y Al-Mharmah, H. (2000). Project management practice by the public sector in a developing country. International Journal of Project Management, 18(2), 105-109.

Ahlemann, F., Teuteberg, F., y Vogelsang, K. (2009). Project management standards - Diffusion and application in Germany and Switzerland. International Journal of Project Management, 27(3), 292-303.

Bryde, D. J. (2003). Project management concepts, methods and application. International Journal of Operations \& Production Management, 23(7), 775-793.

Fortune, J., White, D., Jugdev, K. y Walker, D. (2011). Looking again at current practice in project management. International Journal of Managing Projects in Business, 4(4), 553-572.

Gómez Pérez, Liliana L. (2010). Diseño de una estructura de costos para el procesamiento de espuma flexible de poliuretano en la empresa espumados el oriente de Sogamoso. (Tesis Monográfica, UPTC Seccional Sogamoso).

Ika, L. A., Diallo, A., y Thuillier, D. (2010). Project Management in the international development industry. The project coordinator's perspective. International Journal of Managing Projects in Business, 3(1), 61-93.

Montes-Guerra, M. I., Gimena Ramos, F. N., Pérez-Ezcurdia, M. A., y Díez-Silva, H. M. (2011). Exploring Project Management Bodies of Knowledge and Monitoring \& Control Orientation. In XV International Congress on Project Engineering (Ed.), Asociación Española de Ingeniería de Proyectos - AEIPRO, International Project Management Association - IPMA. Huesca (España).

Murphy, A., y Ledwith, A. (2007). Project management tools and techniques in high-technology SMEs. Management Research News, 30(2), 153-166.

Muriithi, N. y Crawford, L. (2003). Approaches to project management in Africa: Implications for international development projects. International Journal of Project Management, 21(5), 309-319.

Morris, P. W. G., Crawford, L., Hodgson, D., Shepherd, M. M. y Thomas, J. (2006). Exploring the role of formal bodies of knowledge in defining a profession - The case of project management. International Journal of Project Management, 24(8), 710-721.

Project Management Institute PMBOKR. (2012). A guide to the Project Management Body of Knowledge. (PMI Fif5tth EditionEd.). Pennsylvania, USA:. PMI.

Shenhar, A. J., y Dvir, D. (2007). Project Management Research: The Challenge and Opportunity. Project Management Journal, 38(2), 93-99.

White, D. y Fortune, J. (2002). Current practice in project management - an empirical study. International Journal of Project Management, 20(1), 1-11.

Wirth, I. y Tryloff, D. E. (1995). Preliminary comparison of six efforts to document the project-management body of knowledge. International Journal of Project Management, 13(2), 109-118. 